\title{
CT-US fusion imaging increases the feasibility of early ultrasound- guided percutaneous intervention of local drug therapy in pancreatic contusion and laceration
}

\author{
Cong Feng ${ }^{1 \#}$, Libo Wang ${ }^{2 \#}$, Sai Huang ${ }^{3 \#}$, Qinrui Xing ${ }^{4 \#}$, Xuan Zhou ${ }^{1}$, Ning Xing ${ }^{5}$, Faqin Lv $^{6}$, Tanshi Li ${ }^{1}$ \\ ${ }^{1}$ Department of Emergency, First Medical Center, General Hospital of the PLA, Beijing, China; ${ }^{2}$ Department of Ultrasound, Hainan Hospital of the \\ PLA General Hospital, Sanya, China; ${ }^{3}$ Department of Hematology, Fifth Medical Center, General Hospital of the PLA, Beijing, China; ${ }^{4}$ Department \\ of Emergency, Hainan Hospital of the PLA General Hospital, Sanya, China; ${ }^{5}$ Department of Radiology, First Medical Center, General Hospital of \\ the PLA, Beijing, China; ${ }^{6}$ Department of Ultrasound, Third Medical Center, General Hospital of the PLA, Beijing, China \\ Contributions: (I) Conception and design: T Li, F Lv, N Xing; (II) Administrative support: T Li, F Lv, N Xing; (III) Provision of study materials or \\ patients: C Feng, L Wang; (IV) Collection and assembly of data: Q Xing, X Zhou; (V) Data analysis and interpretation: S Huang; (VI) Manuscript \\ writing: All authors; (VII) Final approval of manuscript: All authors. \\ \#These authors contributed equally to this work as co-first authors. \\ Correspondence to: Tanshi Li, MD. Department of Emergency, First Medical Center, General Hospital of the PLA, Beijing 100853, China. \\ Email: 1ts301@163.com; Faqin Lv, MD. Department of Ultrasound, Third Medical Center, General Hospital of the PLA, Beijing 100853, China. \\ Email: lvjin8912@163.com; Ning Xing, MD. Department of Radiology, First Medical Center, General Hospital of the PLA, Beijing 100853, China. \\ Email: xingning0919@hotmail.com.
}

Background: Multimodal fusion imaging (MMFI) was usually used to assist percutaneous procedures for difficult lesions, with most applications occurring with hepatic and prostatic interventions. This paper aimed to evaluate the precision and effectiveness of computed tomography-ultrasound (CT-US) fusion imaging (CUFI)-assisted US-guided percutaneous intervention (UGPI) in early local drug therapy for pancreatic contusion and laceration (PCL).

Methods: A total of 12 pigs with PCL were randomly divided into a CUFI-assisted UGPI (MU) group (n=6) and a single UGPI (SU) group (n=6). The MU group underwent CUFI-assisted UGPI of locally applied medical protein glue $(1 \mathrm{~mL})$ injection while the SU group received the same therapy using two-dimensional UGPI. The duration and accuracy of each procedure were observed in the 2 groups.

Results: In the MU group, the overall time of the procedure for locking the plane was $1.85 \pm 0.06$ minutes. Less time was spent in the selection of the pathway and puncture site in the MU group compared with the SU group $(6.56 \pm 0.42$ vs. $7.61 \pm 0.44$ minutes, $\mathrm{P}<0.01)$. The duration of puncturing and drug injection was also shorter in the MU group than in the SU group $(3.41 \pm 0.30$ vs. $4.20 \pm 0.20$ minutes, $\mathrm{P}<0.01)$ and the MU group had a higher accuracy of medical protein glue injection than the $\mathrm{SU}$ group $(100 \%$ vs. $50 \%, \mathrm{P}<0.05)$.

Conclusions: CUFI could increase the precision and effectiveness of early UGPI in the delivery of local drug therapy in PCL.

Keywords: Multimodal fusion imaging (MMFI); feasibility; ultrasound-guided percutaneous intervention; pancreatic contusion and laceration (PCL)

Submitted Jun 02, 2020. Accepted for publication Nov 06, 2020.

doi: $10.21037 /$ atm-20-4426

View this article at: http://dx.doi.org/10.21037/atm-20-4426 


\section{Introduction}

Although pancreatic trauma is rare, its mortality and morbidity are high (1). Pancreatic contusion and laceration (PCL) is classified as a grade I-II injury based on the American Association for the Surgery of Trauma (AAST) organ injury scaling (2), with a conservative strategy being recommended as its primary form of management (3). Ultrasound (US)-guided percutaneous intervention (UGPI) plays an important role in the clinical management of PCL, as it is used for the delivery of local drug therapy and fluid drainage (4-8). In addition, particular precision in the delivery of UGPI is required in PCL because of the retroperitoneal location of the pancreas.

In comparison to guided approaches using computed tomography (CT) and endoscopic US (EUS), the use of transabdominal US is much faster and cheaper (4). Multimodal fusion imaging (MMFI) is usually used to assist percutaneous procedures for difficult lesions, with most applications occurring with hepatic and prostatic interventions (9). However, very few studies have focused on the application of MMFI techniques for UGPI in pancreatic diseases. Although Zhang et al. have reported on the efficacy of US-CT image fusion guidance in UGPI for acute pancreatitis (10), similar research regarding PCL is still limited.

Fibrin sealants have been used extensively in pancreatic surgery to act as tissue sealants, ideally preventing pancreatic leaks (11-13). Early local protein glue therapy for PCL has been preliminarily observed by our research team (5), but its practical application still requires assistance of minimally invasive procedures with a percutaneous approach. This study aimed to evaluate the precision and effectiveness of CT-US fusion imaging (CUFI)-assisted UGPI in the early management of local drug therapy for PCL.

We present the following article in accordance with the ARRIVE reporting checklist (available at http://dx.doi. org/10.21037/atm-20-4426).

\section{Methods}

\section{Etbics}

The experiments were performed under a project license (No. S2020-045-01) granted by the ethics committee of animal research from the General Hospital of the PLA, in compliance with its guidelines for the care and use of animals.

\section{Experimental animals}

A total of 12 healthy male miniature pigs weighing $10 \pm 1 \mathrm{~kg}$ were provided by the Experimental Animal Center of the PLA General Hospital (Beijing, China).

\section{Drugs and reagents}

Pentobarbital sodium was acquired from the National Pharmaceutical Group Chemical Reagent Co., Ltd. (China) while heparin sodium injection was obtained from the Shanghai First Biochemical Pharmaceutical Co., Ltd. (China). The medical protein glue (EC type), a tissue anatomic adhesive widely used in surgery, was procured from Guangzhou Baiyun Medical Adhesive Co., Ltd. (China).

\section{Main experimental equipment}

A Color Doppler Ultrasound Scanner (LOGIQ E9 XDClear, GE Healthcare, USA) and a 21-gauge percutaneous transhepatic cholangiography (PTC) puncture needle (Bard Biopsy System, Covington, GA, USA) were used.

\section{Experimental groups}

All 12 pigs with PCL were randomly divided into a CUFIassisted UGPI group $(\mathrm{n}=6)(\mathrm{MU})$, and a single UGPI group $(\mathrm{n}=6)(\mathrm{SU})$ and each group received an early medical protein glue $(1 \mathrm{~mL})$ injection. In the $\mathrm{MU}$ group, this was performed under CUFI-assisted UGPI, while in the SU group, this was performed using UGPI without the assistance of other techniques.

\section{Model development}

All pigs were fasted for 12 hours and had no access to water for 4 hours before undergoing surgery. Pigs were anesthetized by intramuscular injection of $3 \%$ pentobarbital sodium $(30 \mathrm{mg} / \mathrm{kg})$. After successful anesthesia, the animals were placed on the bench in the supine position and heparin (300 IU/kg) was given intravenously to resist coagulation.

Following the methods used in our previous procedures for PCL model development (5), we used a surgically exposed pancreas and prepared one site of PCL about $1.5 \mathrm{~cm}$ in length using hemostatic forceps on the surface of the pancreatic body. The depth of the contusion and laceration was made less than half of the thickness of the 

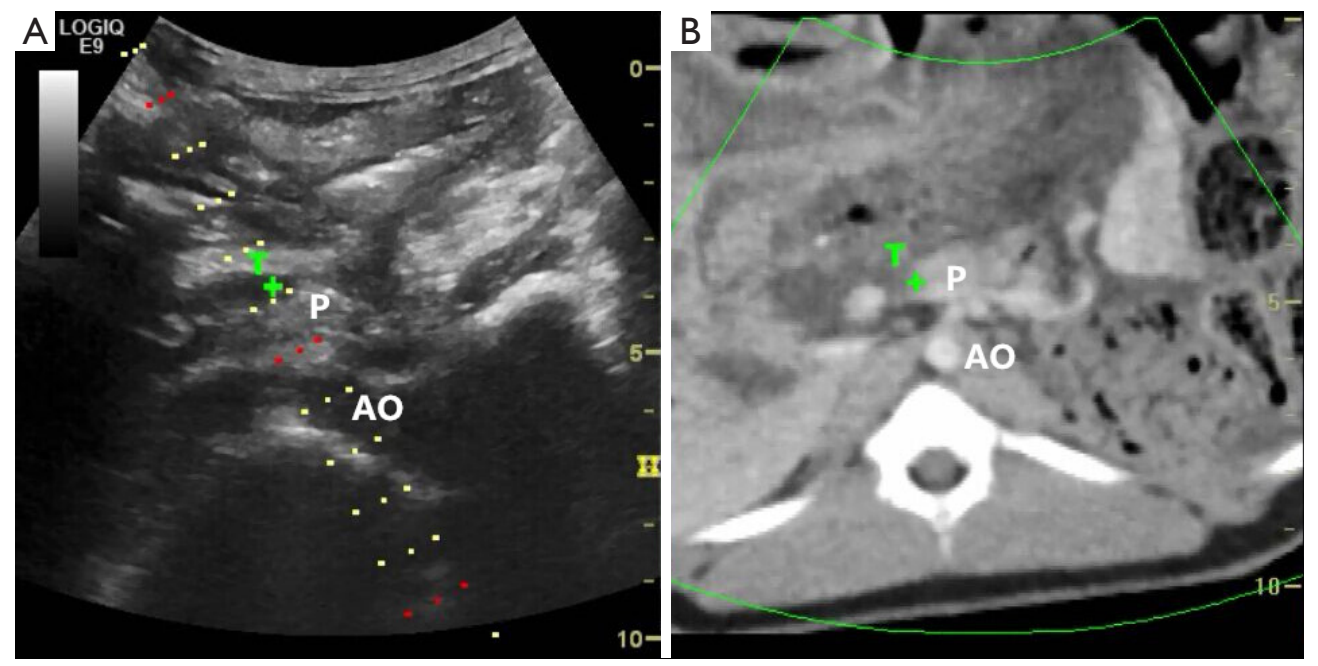

Figure 1 CUFI-assisted UGPI of early local drug therapy on PCL. After identifying the PCL under fusion images, the site of the wound was marked with "T+" using the "Target" function under the volume navigation mode, and the UGPI pathway was also selected. UGPI of early local drug injection was guided along the pathway and marked with "T". CUFI, CT-US fusion imaging; UGPI, ultrasound-guided percutaneous intervention; PCL, pancreatic contusion and laceration; P, pancreas; AO, abdominal aorta.

pancreas to avoid damaging the main duct. The wound of contusion and laceration was rough, the pancreatic capsule was ruptured, and there was persistent bloody fluid exuding from the crack, which met the classification criteria of a scale II injury based on the AAST criteria (2). After the model was successfully constructed for 30 minutes, early local drug therapy under CUFI-assisted UGPI or single UGPI was initiated.

\section{Early local drug therapy in the two groups}

In the MU group, pancreatic enhanced CT volume images with a thickness of $1.5 \mathrm{~mm}$ for fusion were acquired following the model construction. After CT images were imported into the ultrasound scanner, both ultrasound and CT images could be displayed on the same screen simultaneously under the volume navigation mode. Once the multimodality images were fused, the position of the animal body was maintained, and the acoustic beam of the probe was directed perpendicular to the abdomen. The bifurcation of the celiac trunk from the aorta was then marked on the US image, although the intrahepatic portal vein, hepatic vein bifurcation, celiac trunk, or origin of the renal artery could also be chosen as the lock point. This was labeled as the same start site of the celiac trunk on the CT image and matched with a US image with a similar spatial position. The fusion images were finely adjusted to achieve the perfect fusion effect by using the "OVERLAY" function under the volume navigation mode. After identifying the PCL under fusion images, the site of the wound was marked with "T+" using the "Target" function (Figure 1). After fusion of the images, the CUFI-assisted UGPI pathway was selected and displayed on the US image (Figure 1). Subsequently, the UGPI of the medical protein glue $(1 \mathrm{~mL})$ injection to the PCL wound was implemented along the pathway and directed by the "T+" mark. In the SU group, the pigs underwent the UGPI of the medical protein glue $(1 \mathrm{~mL})$ injection using two-dimensional US images only (Figure 2).

\section{Outcome measures}

The times required for selecting the pathway to the puncture site and in puncturing and drug injection were recorded and compared between the two groups. The puncture needle was removed after the medical protein glue injection and the pancreas was surgically exposed again to determine the accuracy of drug injection in the 2 groups. If more than $80 \%$ of each wound was covered with protein glue, the procedure was deemed accurate. Any related injuries to other organs by UGPI under the 2 different methods were also examined. The investigator who performed the UGPI was blinded to the model development. 

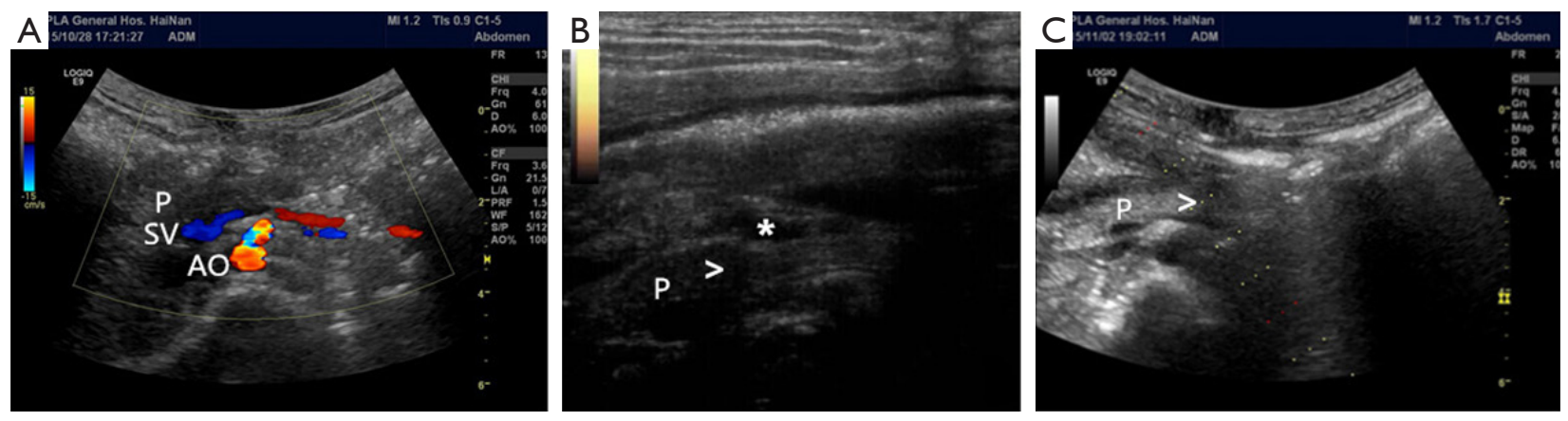

Figure 2 The observation of PCL under two-dimensional ultrasound images of B-mode ultrasound and contrast-enhanced ultrasound. (A) A normal pancreas under two-dimensional ultrasound images (P, pancreas; SV, splenic vein; AO, abdominal aorta); (B) PCL was identified under B-mode ultrasound, with the pancreatic body ("P"), PCL (">”), and a small amount of effusion around the pancreas being visible ("**); (C) in the SU group, the UGPI of the medical protein glue $(1 \mathrm{~mL})$ injection depended only on two-dimensional ultrasound images. PCL, pancreatic contusion and laceration; UGPI, ultrasound-guided percutaneous intervention; SU, single UGPI.

\section{Statistical analysis}

Continuous variables are expressed as the mean (SD) and were analyzed by unpaired $t$-test or the Wilcoxon rank-sum test, as appropriate. Categorical variables are expressed as absolute (n) and relative (\%) frequency and were analyzed by the chi-square test or Fisher's exact test as appropriate. A $P$ value $<0.05$ indicated statistical difference, while a $P$ value $<0.01$ indicated a statistically significant difference.

\section{Results}

All 12 pigs were identified as having a PCL which manifested as a low-enhancement area, irregular shape, and blurred boundary of the contusion and laceration next to the area of the normal pancreas. The depth of the PCL was less than $50 \%$ of the pancreatic thickness at the same site (Figure 2).

In the MU group, the overall duration of the procedure for locking the plane (finding the vascular bifurcation and fixing the "OVERLAY") was $1.85 \pm 0.06$ minutes, while the time required for pathway and puncture site selection was shorter in the MU group than in the SU group $(6.56 \pm 0.42 v s$. $7.61 \pm 0.44$ minutes, $\mathrm{P}<0.01)$. The duration of puncturing and drug injection was also less in the MU group compared with the $\mathrm{SU}$ group $(3.41 \pm 0.30$ vs. $4.20 \pm 0.20$ minutes, $\mathrm{P}<0.01)$. The details of the timing for each animal can be found in Table S1.

In the SU group, 3 out of 6 PCL wounds were successfully covered by medical protein glue indicating an accuracy of $50 \%$. In the MU group, the accuracy was significantly improved, with all 6 (100\% accuracy) of the PCL wounds covered by medical protein glue. The accuracy of the 2 groups was statistically different $(100 \%$ vs. $50 \%, \mathrm{P}<0.05)$.

The observation of laparotomy revealed no related injuries to other vital organs or tissues in either group.

\section{Discussion}

While pancreatic trauma accounts for less than $2 \%$ of blunt trauma cases, it is associated with considerably high morbidity and mortality in cases of delayed treatment (14). PCL is a type of pancreatic trauma without main pancreatic duct injury and is classified as grade I or II based on the AAST classification criteria (2). Although conservative management is usually advocated for PCL, secondary traumatic pancreatitis or rupture of the pancreatic duct has a mortality rate of up to $2,045 \%(15-18)$. We have observed that local drug therapy in the early stage could effectively control the development of PCL (5), but its practical application calls for more precise and effective minimally invasive procedures when a percutaneous approach is used.

CT is commonly used as the first-choice imaging modality for the diagnosis and minimally invasive intervention in pancreatic trauma cases (19). CT guidance of the percutaneous approach has been demonstrated to accurately determine the puncture point, pathways, and depth, and is thus widely applicable to retroperitoneal diseases $(20,21)$. However, compared to CT guidance, UGPI can be performed at the bedside, guarantee a real-time image, and be applied to complicated and serious cases (4) although its use is strongly dependent on operator expertise, in particular for the early management of pancreatic trauma (22). The usefulness of guided approaches for interventional procedures in pancreatic 
disease by CT and EUS has been reported, and although transabdominal US was found to be faster and cheaper, its application in the management of PCL was limited (4).

MMFI can provide a real-time fusion image of twodimensional US with CT or other modal of radiology image, such as PET-CT or MRI $(23,24)$. This technique has many advantages as a form of interventional ultrasound since it can match the characteristics of two different types of imaging in a single examination and provide additional anatomical, functional, and metabolic information during US-guided procedures $(9,25)$. CUFI is commonly used to assist percutaneous procedures for organs which are challenging to view, such as the pancreas which, because of its retroperitoneal location, is easily hidden or distorted by other tissues and gases in the abdominal cavity. Thus far, only a few studies have reported on the potential usefulness of CUFI-assisted UGPI for acute pancreatitis and other pancreatic diseases $(10,26,27)$, and similar research on whether or not CUFI can provide safer and more effective guidance for early PCL interventions are also rare.

In this study, we observed the precision and effectiveness of CUFI-assisted UGPI in the early administration of local drug therapy for PCL based on our preliminary study and achieved remarkable results. The time required for therapy was significantly reduced while the accuracy for early UGPI was greater in the MU group compared with the SU group. The wound site of PCL could be marked and more easily guided on fusion images during UGPI with the help of CUFI. Therefore, CUFI could increase the feasibility of early UGPI on PCL and serve ideally in the minimally invasive management of local drug therapy. In further studies on the use of local drug therapy, radio-opaque glue and simple post-treatment CT scans may be used to evaluate the accuracy of CUFI-assisted UGPI.

\section{Conclusions}

CUFI could increase the precision and effectiveness of early UGPI for local drug therapy on PCL.

\section{Acknowledgments}

Funding: The present study was supported by grants from the National Natural Science Fund (No. 81701961, 82072200), the PLA Logistic Major Science and Technology Project (16QNP135), the Beijing Science and Technology New Star Project (No. XX2018019/Z181100006218028), the PLA General Hospital Science and Technology Project
(No. 16KMM56, 2017FC-WJFWZX-30, and 2018XXFC20), the Winter Olympics of Science and Technology (No. 2019YFF0302300), and the $13^{\text {th }}$ Five-year Plan For The Key Discipline Construction Project Of The PLA (No. A350109).

\section{Footnote}

Reporting Checklist: The authors have completed the ARRIVE reporting checklist. Available at http://dx.doi. org/10.21037/atm-20-4426

Data Sharing Statement: Available at http://dx.doi. org/10.21037/atm-20-4426

Peer Review File: Available at http://dx.doi.org/10.21037/ atm-20-4426

Conflicts of Interest: All authors have completed the ICMJE uniform disclosure form (available at http://dx.doi. org/10.21037/atm-20-4426). The authors have no conflicts of interest to declare.

Etbical Statement: The authors are accountable for all aspects of the work in ensuring that questions related to the accuracy or integrity of any part of the work are appropriately investigated and resolved. Experiments were performed under a project license (No. S2020-045-01) granted by the ethics committee of animal research from the General Hospital of the PLA, in compliance with its guidelines for the care and use of animals.

Open Access Statement: This is an Open Access article distributed in accordance with the Creative Commons Attribution-NonCommercial-NoDerivs 4.0 International License (CC BY-NC-ND 4.0), which permits the noncommercial replication and distribution of the article with the strict proviso that no changes or edits are made and the original work is properly cited (including links to both the formal publication through the relevant DOI and the license). See: https://creativecommons.org/licenses/by-nc-nd/4.0/.

\section{References}

1. Soreide K, Weiser TG, Parks RW. Clinical update on management of pancreatic trauma. HPB (Oxford) 2018;20:1099-108.

2. Moore EE, Cogbill TH, Malangoni MA, et al. Organ injury scaling, II: Pancreas, duodenum, small bowel, colon, 
and rectum. J Trauma 1990;30:1427-9.

3. Ho VP, Patel NJ, Bokhari F, et al. Management of adult pancreatic injuries: A practice management guideline from the Eastern Association for the Surgery of Trauma. J Trauma Acute Care Surg 2017;82:185-99.

4. D'Onofrio M, Beleu A, De Robertis R. Ultrasound-guided percutaneous procedures in pancreatic diseases: new techniques and applications. Eur Radiol Exp 2019;3:2.

5. Feng C, Yang H, Huang S, et al. Early local drug therapy for pancreatic contusion and laceration. Pancreatology 2019;19:285-9.

6. Tang J, Lv F, Li W, et al. Percutaneous injection of hemostatic agents for severe blunt hepatic trauma: an experimental study. Eur Radiol 2008;18:2848-53.

7. Song Q, Tang J, Lv FQ, et al. Evaluation of blunt pancreatic injury with contrast-enhanced ultrasonography in comparison with contrast-enhanced computed tomography. Exp Ther Med 2013;5:1461-5.

8. Lv F, Tang J, Luo Y, et al. Percutaneous treatment of blunt hepatic and splenic trauma under contrast-enhanced ultrasound guidance. Clin Imaging 2012;36:191-8.

9. Paparo F, Piccazzo R, Cevasco L, et al. Advantages of percutaneous abdominal biopsy under PET-CT/ ultrasound fusion imaging guidance: a pictorial essay. Abdom Imaging 2014;39:1102-13.

10. Zhang H, Chen GY, Xiao L, et al. Ultrasonic/CT image fusion guidance facilitating percutaneous catheter drainage in treatment of acute pancreatitis complicated with infected walled-off necrosis. Pancreatology 2018;18:635-41.

11. Mita K, Ito H, Fukumoto M, et al. Pancreaticojejunostomy using a fibrin adhesive sealant (TachoComb) for the prevention of pancreatic fistula after pancreaticoduodenectomy. Hepatogastroenterology 2011;58:187-91.

12. Satoi S, Toyokawa H, Yanagimoto H, et al. Reinforcement of pancreticojejunostomy using polyglycolic acid mesh and fibrin glue sealant. Pancreas 2011;40:16-20.

13. Mita K, Ito H, Fukumoto $M$, et al. A fibrin adhesive sealing method for the prevention of pancreatic fistula following distal pancreatectomy. Hepatogastroenterology 2011;58:604-8.

14. Dave S, London S. Pancreatic Trauma. StatPearls. Treasure Island (FL): StatPearls Publishing LLC.; 2019.

15. Petrone P, Moral Alvarez S, Gonzalez Perez M, et al. Pancreatic trauma: Management and literature review. Cir Esp 2017;95:123-30.

16. Soreide K. Pancreas injury: the good, the bad and the ugly. Injury 2015;46:827-9.

17. Siboni S, Kwon E, Benjamin E, et al. Isolated blunt pancreatic trauma: A benign injury? J Trauma Acute Care Surg 2016;81:855-9.

18. Debi U, Kaur R, Prasad KK, et al. Pancreatic trauma: a concise review. World J Gastroenterol 2013;19:9003-11.

19. Iacobellis F, Laccetti E, Tamburrini S, et al. Role of multidetector computed tomography in the assessment of pancreatic injuries after blunt trauma: a multicenter experience. Gland Surg 2019;8:184-96.

20. Rekhi S, Anderson SW, Rhea JT, et al. Imaging of blunt pancreatic trauma. Emerg Radiol 2010;17:13-9.

21. Baudin G, Chassang M, Gelsi E, et al. CT-guided percutaneous catheter drainage of acute infectious necrotizing pancreatitis: assessment of effectiveness and safety. AJR Am J Roentgenol 2012;199:192-9.

22. Sorrentino L, Chiara O, Mutignani M, et al. Combined totally mini-invasive approach in necrotizing pancreatitis: a case report and systematic literature review. World J Emerg Surg 2017;12:16.

23. Ewertsen C, Saftoiu A, Gruionu LG, et al. Real-time image fusion involving diagnostic ultrasound. AJR Am J Roentgenol 2013;200:W249-55.

24. Mauri G, Gennaro N, De Beni S, et al. Real-Time US-(18) FDG-PET/CT Image Fusion for Guidance of Thermal Ablation of (18)FDG-PET-Positive Liver Metastases: The Added Value of Contrast Enhancement. Cardiovasc Intervent Radiol 2019;42:60-8.

25. Paparo F, Massollo M, Rollandi L, et al. The clinical role of multimodality imaging in the detection of prostate cancer recurrence after radical prostatectomy and radiation therapy: past, present, and future. Ecancermedicalscience 2015;9:570.

26. Sofuni A, Itoi T, Itokawa F, et al. Real-time virtual sonography visualization and its clinical application in biliopancreatic disease. World J Gastroenterol 2013;19:7419-25.

27. Sumi H, Itoh A, Kawashima H, et al. Preliminary study on evaluation of the pancreatic tail observable limit of transabdominal ultrasonography using a position sensor and CT-fusion image. Eur J Radiol 2014;83:1324-31.

(English Language Editor: B. Draper; Quality Control Editor: J. Gray)

Cite this article as: Feng C, Wang L, Huang S, Xing Q, Zhou X, Xing N, Lv F, Li T. CT-US fusion imaging increases the feasibility of early ultrasound-guided percutaneous intervention of local drug therapy in pancreatic contusion and laceration. Ann Transl Med 2021;9(4):307. doi: 10.21037/atm20-4426 
Supplementary

Table S1 The timing of the procedures for each pig separately

\begin{tabular}{lcc}
\hline Pigs' marks & The time of selecting the pathway and puncture site (minutes) & The time of puncturing and drug injection (minutes) \\
\hline MU group & 6.33 & 3.25 \\
P1 & 6.75 & 3.67 \\
P2 & 7 & 3 \\
P3 & 6.67 & 3.83 \\
P4 & 6.75 & 3.33 \\
P5 & 5.83 & 3.37 \\
P6 & & \\
SU group & 8.25 & 4.33 \\
P7 & 7.33 & 4 \\
P8 & 7.15 & 4 \\
P9 & 7.25 & 4.25 \\
P10 & 7.67 & 4.15 \\
P11 & 8 & 4.5 \\
P12 & & \\
\hline
\end{tabular}

